\title{
A RESTRICTION THEOREM FOR MODULES HAVING A SPHERICAL SUBMODULE
}

\author{
NICOLAS ANDRUSKIEWITSCH AND JUAN A. TIRAO
}

\begin{abstract}
We introduce the following notion: a finite dimensional representation $V$ of a complex reductive algebraic group $G$ is called spherical of rank one if the generic stabilizer $M$ is reductive, the pair $(G, M)$ is spherical and $\operatorname{dim} V^{M}=1$. Let $U$ be another finite dimensional representation of $G$; we denote by $S^{\prime}(U)\left(S^{\prime}(U)^{G}\right)$ the ring of polynomial functions on $U$ (the ring of $G$-invariant polynomial functions on $U$ ). We characterize the image of $S^{\prime}(U \oplus V)^{G}$ under the restriction map into $S^{\prime}\left(U \oplus V^{M}\right)$ as the $W=N_{G}(M) / M$ invariants in the Rees ring associated to an ascending filtration of $S^{\prime}(U)^{M}$. Furthermore, under some additional hypothesis, we give an isomorphism between the graded ring associated to that filtration and $S^{\prime}(U)^{P}$, where $P$ is the stabilizer of an unstable point whose $G$-orbit has maximal dimension.
\end{abstract}

\section{INTRODUCTION}

Let $\mathfrak{g}_{\mathbb{R}}=\mathfrak{k}_{\mathbb{R}} \oplus \mathfrak{p}_{\mathbb{R}}$ be a Cartan decomposition of a real semisimple Lie algebra $\mathfrak{g}_{\mathbb{R}}$ and let $\mathfrak{g}=\mathfrak{k} \oplus \mathfrak{p}$ be the corresponding complexification. Let $\theta$ be the associated Cartan involution. Also let $\mathfrak{a}_{\mathbb{R}}$ be a maximal abelian subspace of $\mathfrak{p}_{\mathbb{R}}$ and let $\mathfrak{a}$ be its complexification. Now let $K$ be the analytic subgroup of the adjoint group of $\mathfrak{g}$ with Lie algebra $\operatorname{ad}_{\mathfrak{g}}(\mathfrak{k})$. Also let $M$ be the centralizer of $\mathfrak{a}$ in $K$ and let $W$ be the Weyl group associated to $(\mathfrak{g}, \mathfrak{a})$, i.e., $W=N_{K}(M) / M$, where $N_{L}(S)$ is the notation for the normalizer in $L$ of $S$.

If $V$ is any finite-dimensional complex vector space, let $S^{\prime}(V)$ be the ring of all polynomial functions on $V$. The well-known Chevalley Restriction Theorem states that the restriction homomorphism $S^{\prime}(\mathfrak{p}) \rightarrow S^{\prime}(\mathfrak{a})$ maps $S^{\prime}(\mathfrak{p})^{K}$ isomorphically onto $S^{\prime}(\mathfrak{a})^{W}$. (Here $V^{L}$ denotes the submodule of an $L$-module $V$ consisting of all $L$-invariants.) This theorem was generalized by Luna and Richardson [LR]:

Let $G$ be a reductive complex algebraic group acting linearly (and morphically) on a finite-dimensional vector space $U$ and assume that $(U, G)$ has generically closed orbits; i.e., the union of all closed orbits contains a nonempty Zariski open subset of $U$. Pick any $x \in U$ such that the orbit $G x$ is closed and $G^{x}$ is conjugated to $G^{y}$ for all $y$ in an open neighborhood of $x$. The conjugacy class of the isotropy subgroup $M=G^{x}$ is called a principal isotropy class. The generalization of the Chevalley Restriction Theorem given in [LR] states that the restriction map $S^{\prime}(U) \rightarrow S^{\prime}\left(U^{M}\right)$ maps $S^{\prime}(U)^{G}$ isomorphically

Received by the editors December 15, 1988 and, in revised form, March 9, 1990.

1980 Mathematics Subject Classification (1985 Revision). Primary 14D25, 14L30.

This work was supported by CONICET, CONICOR, and FAMAF. 
onto $S^{\prime}\left(U^{M}\right)^{W}$, where $W=N_{G}(M) / M$. (A word of caution: this is not a generalization strictu senso because $\mathfrak{a} \neq \mathfrak{p}^{M}$ in general. The Chevalley Restriction Theorem mentioned in [LR] is the version " $\mathfrak{g}_{\mathbb{R}}$ of type II", in Cartan's terminology).

However, although the Chevalley Restriction Theorem and its generalization are quite powerful tools, they have a restricted field of applications: indeed, almost every representation of a semisimple group has trivial principal isotropy class (see $[\mathrm{AP}]$ ).

Now let $G$ act linearly on another finite-dimensional vector space $N$. Then the restriction map $S^{\prime}(N \oplus U) \rightarrow S^{\prime}\left(N \oplus U^{M}\right)$ induces a monomorphism $S^{\prime}(N \oplus$ $U)^{G} \rightarrow S^{\prime}\left(N \oplus U^{M}\right)^{N_{G}(M)}$ whose image seems to be very difficult to characterize. A first step in this direction was given by Tirao in [T] for the following case: $G=K, U=\mathfrak{p}, N=\mathfrak{k}$ and $\operatorname{dim} \mathfrak{a}=1$. The proof given there is geometric and uses a one-parameter subgroup suitably chosen. Another proof can be found in [A1] and one of the aims of this article is to present a generalization of this fact, inspired by this second proof.

We make the following additional hypothesis on $(U, G):($ a) The pair $(G, M)$ is a spherical pair (sometimes called a Gelfand pair); (b) $\operatorname{dim} U^{M}=1$. Then we say that $(U, G)$ is a spherical representation of rank one.

In this case, the image of the restriction map for any $N$ is characterized, as in Tirao's case, by

$$
\left(\bigoplus_{n \in \mathbb{N}_{0}}\left(\bigoplus_{\gamma \in \Gamma_{n}} S^{\prime}(N)_{\gamma}^{M} \otimes S_{n}^{\prime}\left(U^{M}\right)\right)\right)^{W}
$$

Here $S_{n}^{\prime}$ is the subspace of all homogeneous polynomials of degree $n$ and

$$
\Gamma_{n}=\left\{\gamma \in G^{\wedge}: \gamma^{M} \neq 0, m(\gamma) \leq n\right\},
$$

where $m(\gamma)$ is the degree of homogeneity of $\gamma^{*}$ in the harmonic polynomials in $U$.

A further generalization is found if $(U, G)$ is a "product" of spherical representations of rank one (see also [A2]). Moreover,

$$
C_{n}=\bigoplus_{\gamma \in G^{\wedge}} S_{m(\gamma) \leq n} S^{\prime}(N)_{\gamma}^{M},
$$

defines a filtration of $C=S^{\prime}(N)^{M}$.

The second purpose of this article is to characterize the graded ring associated to this filtration as the ring of $P$-invariants in $S^{\prime}(N)$ for a suitable subgroup $P$ of $G$. In the case: $G=K, U=\mathfrak{p}, N=\mathfrak{k}, \operatorname{dim} \mathfrak{a}=1$ and $P$ is the isotropy subgroup of a principal nilpotent element in $\mathfrak{p}$, this was obtained by Tirao (unpublished) using ideas in the spirit of the proof given in [T]. The proof depends on the existence of a suitable $z \in \mathfrak{k}$. Our proof, however, avoids this and it is available for the general case under some additional conditions. We also remark that one can not expect in general that $P$ contains a maximal unipotent subgroup of $G$; for example, this is false when $\mathfrak{g}_{\mathbb{R}}=\mathfrak{s p}(n, 1)$, although it is true when $\mathfrak{g}_{\mathbb{R}}=\mathfrak{s o}(n, 1)$ or $\mathfrak{g}_{\mathbb{R}}=\mathfrak{s u}(n, 1)$.

This theorem in some sense reduces the study of $S^{\prime}(U \oplus N)^{G}$ to the study of $S^{\prime}(N)^{P}$. For example, $S^{\prime}(U \oplus N)^{G}$ is a polynomial ring if and only if $S^{\prime}(N)^{P}$ is a polynomial ring. 
Finally, we give some applications; for example, we compute explicitly a presentation of the ring $S^{\prime}(\mathfrak{g})^{K}$ when $\mathfrak{g}_{\mathbb{R}}=\mathfrak{s p}(2,1)$.

\section{Preliminaries}

As usual, $L^{\wedge}$ denotes the set of equivalence classes of finite dimensional irreducible representations of an algebraic reductive complex linear group $L$. We will identify $\tau \in L^{\wedge}$ with the space on which $L$ acts. We will exploit the following well-known version of the

Schur Lemma. For $\tau, \lambda \in L^{\wedge}, \operatorname{dim}(\tau \otimes \lambda)^{L}=1$ if $\tau=\lambda^{*}, 0$ otherwise.

If $E$ is any $L$-module, and $\tau \in L^{\wedge}$, we denote by $E_{\tau}$ the isotypic component of type $\tau$.

Let us recall briefly the notion of a spherical pair. Let $G$ be a reductive connected algebraic group and $H$ a closed subgroup, over an algebraically closed field of characteristic zero. $(G, H)$ is a spherical pair if it satisfies one of the following equivalent conditions:

(i) $H$ has an open orbit in the flag variety of $G$.

(ii) $H$ has a finite number of orbits in the flag variety of $G$.

(iii) Let $Z$ be an algebraic $G$-variety and let $z \in Z^{H}$; then $G$ has a finite number of orbits in the closure of $G z$.

(iv) Let $\chi$ be a one-dimensional representation of $H$ and let $X$ be the representation of $G$ induced by $\chi$ (i.e., the space of global sections of the associated line bundle over $G / H)$. Then for every $\gamma \in G^{\wedge}, \operatorname{dim}_{\operatorname{Hom}_{G}}(\gamma, X) \leq$ 1 .

(See [BLV] for the history of this result.) It follows from Frobenius reciprocity that (iv) can be also stated as follows: for every $\gamma \in G^{\wedge}$,

$$
\operatorname{dim} \operatorname{Hom}_{H}(\chi, \gamma) \leq 1
$$

viewing $\gamma$ as an $H$-module. In particular, taking $\chi$ trivial, $\operatorname{dim} \gamma^{H} \leq 1$ for all $\gamma \in G^{\wedge}$. On the other hand this implies the above conditions, whenever $H$ is reductive (see $[\mathrm{VK}])$.

Let $G$ now be a reductive complex linear algebraic group, $U$ a finitedimensional $G$-module. Recall that $(U, G)$ is cofree if $S^{\prime}(U)$ is a $S^{\prime}(U)^{G}$ free module. In such a case, $S^{\prime}(U)^{G}$ is a polynomial ring (i.e., $(U, G)$ is coregular) and

$$
S^{\prime}(U)=S^{\prime}(U)^{G} \otimes H
$$

where $\otimes$ is given by multiplication and $H$ is a homogeneous $G$-submodule of $S^{\prime}(U)$.

Let $M$ be a principal isotropy group of $(U, G)$ (i.e., the stabilizer of a point in a closed orbit whose conjugacy class is minimal) and let $A=U^{M}$. Put $W=N_{G}(M) / M$. We know from [LR] that $S^{\prime}(U)^{G} \simeq S^{\prime}(A)^{W}$, via the restriction homomorphism.

Lemma 1 (See also [Po]). Assume that $(U, G)$ has generically closed orbits and that $\operatorname{dim} A=1$. Then $(U, G)$ is cofree and $W$ is a finite cyclic group.

Proof. Clearly, $\operatorname{dim} A / W$ cannot be zero. (The unique closed orbit would be open.) Thus $\operatorname{dim} A / W=1$. By [LR] (see the proof of Theorem 4.2) $(A, W)$ has generically closed orbits. So we have

$$
\operatorname{dim} A / W=1=\operatorname{dim} A-\operatorname{dim} W .
$$


Hence $W$ is finite. It is "contained" in $\mathrm{GL}(1, \mathbb{C})$; so it is cyclic and $(U, G)$ is coregular. Now $\operatorname{codim} \pi^{-1}(\zeta) \leq 1$ for every $\zeta \in U / G$; it cannot be 0 , so $(U, G)$ is cofree. (See for example, [Sch, §4.3].)

Definition. We say that $(U, G)$ is a spherical representation of rank one if it has generically closed orbits, $\operatorname{dim} A=1$, and for all $\rho \in G^{\wedge}, \operatorname{dim} \rho^{M} \leq 1$, where $M$ is a principal isotropy group of $(U, G)$. In particular, $(U, G)$ is irreducible.

Remark 1 . There exists a pair $(V, L)$, where $L$ is a simple connected algebraic group, having generically closed orbits and such that:

(i) If $M$ is in the principal isotropy class, $V^{M}$ is a line but

(ii) $(L, M)$ is not a spherical pair.

Indeed, take $L=A_{6}, V$ the irreducible representation of highest weight $\varphi_{3}$. From [E, Table 1] we know that the generic stabilizer is of type $G_{2}$ and hence (see for example [Po2] or [LV]) $(V, L)$ has generically closed orbits. Moreover, $\operatorname{dim} V^{M}=1$ [E, Table 1]. One the other hand, $\left(A_{6}, G_{2}\right)$ is not a spherical pair, as follows from Kramer's table [VK]. In fact, the intersection of Elashvili's and Kramer's tables gives us all the spherical representations of rank one of simple groups.

Remark 2. From Lemma $1,(U, G)$ is cofree. Let $H$ be as above; then the multiplicity of $\rho$ in $H$ is $\leq 1$. (See [Sch, §4.3].)

Definition. We will say that $(U, G)$ is a spherical representation (of rank $s$ ) if $U=U_{1} \oplus \cdots \oplus U_{s}, G=G_{1} \times \cdots \times G_{s}$, each $U_{i}$ is a $G_{i}$-spherical module of rank one and $G$ acts on $U$ via

$$
\left(k_{1}, \ldots, k_{s}\right)\left(v_{1}, \ldots, v_{s}\right)=\left(k_{1} v_{1}, \ldots, k_{s} v_{s}\right) .
$$

Note that it has generically closed orbits.

\section{THE RESTRICTION THEOREM}

Now assume that $(U, G)$ is a spherical representation of rank one, $S^{\prime}(U)=$ $S^{\prime}(U)^{G} \otimes H$ and let $\Gamma=\left\{\rho \in G^{\wedge}: \rho^{M} \neq 0\right\}$. Then $H=\bigoplus_{\rho \in \Gamma} H_{\rho}$. Moreover, $H_{\rho}$ is irreducible and homogeneous. Clearly, $\rho \in \Gamma \Rightarrow \rho^{*} \in \Gamma$. So we put

$$
m(\gamma)=\text { degree of homogeneity of } H_{\gamma^{*}}, \quad \gamma \in \Gamma \text {. }
$$

Now let $(U, G)=\left(U_{1}, G_{1}\right) \oplus \cdots \oplus\left(U_{s}, G_{s}\right)$ be a spherical representation, where $\left(U_{i}, G_{i}\right)$ are spherical representations of rank one. We introduce the following notation:

$$
\begin{array}{ll}
A=A_{1} \oplus \cdots \oplus A_{s}, & \Gamma=\Gamma_{1} \times \cdots \times \Gamma_{s}, \\
H=H_{1} \otimes \cdots \otimes H_{s}, & M=M_{1} \times \cdots \times M_{s},
\end{array}
$$

where $M_{i}, \Gamma_{i}, A_{i}, H_{i}$ correspond to $\left(U_{i}, G_{i}\right)$.

Recall now that $G^{\wedge}$ identifies with $G_{1}^{\wedge} \times \cdots \times G_{s}^{\wedge}$. Thus $\gamma \in \Gamma$ if and only if $\gamma$ appears in $H$; and in such a case, it does with multiplicity one. Moreover, let us consider the $\mathbb{N}_{0}^{s}$-grading in $S^{\prime}(U)$ given by the decomposition $U=U_{1} \oplus \cdots \oplus U_{s}$. Therefore, $H$ is an $\mathbb{N}_{0}^{s}$-graded $G$-submodule of $S^{\prime}(U)$ and for $\gamma=\gamma_{1} \otimes \cdots \otimes \gamma_{s} \in \Gamma, \gamma^{*}$ appears only in $H_{m(\gamma)}$, where $m(\gamma)=$ $\left(m\left(\gamma_{1}\right), \ldots, m\left(\gamma_{s}\right)\right) \in \mathbb{N}_{0}^{s}$ and $m\left(\gamma_{i}\right)$ corresponds to $\left(U_{i}, G_{i}\right), \gamma_{i}$. 
Clearly, $M$ is a principal isotropy group of $(U, G)$ and $A=U^{M}$. Let $W=$ $N_{G}(M) / M$. We consider the order in $\mathbb{N}_{0}^{s}$ given by $\left(a_{1}, \ldots, a_{s}\right) \leq\left(b_{1}, \ldots, b_{s}\right)$ iff $a_{i} \leq b_{i}$ for all $i=1, \ldots, s$ and we set

$$
\Gamma_{r}=\{\gamma \in \Gamma: m(\gamma) \leq r\},
$$

for every $r \in \mathbb{N}_{0}^{s}$.

Theorem 1. Keep the notations and the hypothesis as above. Let $N$ be a finitedimensional G-module; then the restriction from $N \oplus U$ to $N \oplus A$ induces an isomorphism $\sigma$ of $S^{\prime}(N \oplus U)^{G}$ onto

$$
\left(\bigoplus_{r \in \mathbb{N}_{0}^{s}}\left(\bigoplus_{\gamma \in \Gamma_{r}} S^{\prime}(N)_{\gamma}^{M} \otimes S_{r}^{\prime}(A)\right)\right)^{W}
$$

Proof. The injectivity follows from [LR], Lemma 3.5.

If $E_{1}, E_{2}$ are finite dimensional $L$-modules, the latter trivial, then $\left(E_{1} \otimes E_{2}\right)^{L}=E_{1}^{L} \otimes E_{2}$. Thus

$$
\begin{aligned}
S^{\prime}(N \oplus U)^{G} & =\left(S^{\prime}(N) \otimes S^{\prime}(U)\right)^{G} \\
& =\left(S^{\prime}(N) \otimes S^{\prime}(U)^{G} \otimes H\right)^{G} \\
& =S^{\prime}(U)^{G} \otimes\left(S^{\prime}(N) \otimes H\right)^{G} \\
& =S^{\prime}(U)^{G} \otimes\left(\bigoplus_{\lambda \in \Gamma}\left(S^{\prime}(N)_{\lambda} \otimes H_{\lambda^{*}}\right)^{G}\right) \\
& \stackrel{\sigma}{\rightarrow} S^{\prime}(A)^{W} \otimes\left(\bigoplus_{\lambda \in \Gamma} \sigma\left(S^{\prime}(N)_{\lambda} \otimes H_{\lambda^{*}}\right)^{G}\right) .
\end{aligned}
$$

Let us look more closely at $\left(S^{\prime}(N)_{\lambda} \otimes H_{\lambda^{*}}\right)^{G}$. In general, if $S^{\prime}(N)_{\lambda}=$ $\bigoplus_{i} T_{i}, T_{i} \simeq \lambda$, then $\left(S^{\prime}(N)_{\lambda} \otimes H_{\lambda^{*}}\right)^{G}=\bigoplus_{i}\left(T_{i} \otimes H_{\lambda^{*}}\right)^{G}$. But $\sigma$ is $1-1$ and

$$
\operatorname{dim}\left(T_{i} \otimes H_{\lambda^{*}}\right)^{G}=\operatorname{dim}\left(T_{i}\right)^{M}=1 .
$$

So

$$
\sigma\left(S^{\prime}(N)_{\lambda} \otimes H_{\lambda^{*}}\right)^{G}=S^{\prime}(N)_{\lambda}^{M} \otimes S_{m(\lambda)}^{\prime}(A) .
$$

Hence

$$
\sigma\left(S^{\prime}(N \oplus U)^{G}\right)=S^{\prime}(A)^{W} \otimes\left(\bigoplus_{\lambda \in \Gamma} S^{\prime}(N)_{\lambda}^{M} \otimes S_{m(\lambda)}^{\prime}(A)\right) .
$$

Therefore, we have

$$
\begin{aligned}
\sigma\left(S^{\prime}(N \oplus U)^{G}\right) & =\left(\bigoplus_{n \in \mathbb{N}_{0}^{s}} S_{n}^{\prime}(A) \otimes\left(\bigoplus_{\lambda \in \Gamma} S^{\prime}(N)_{\lambda}^{M} \otimes S_{m(\lambda)}^{\prime}(A)\right)\right)^{W} \\
& =\left(\bigoplus_{n \in \mathbb{N}_{0}^{s}}\left(\bigoplus_{\lambda \in \Gamma_{n}} S^{\prime}(N)_{\lambda}^{M} \otimes S_{n}^{\prime}(A)\right)\right)^{W} \cdot \square
\end{aligned}
$$


Remark 3. The theorem remains true if we replace $N$ by any affine variety on which $G$ acts.

\section{THE ASCENDING FILTRATION}

Now let $(U, G)$ be a spherical representation of rank one, $X$ an irreducible $G$-variety and $M$ a principal isotropy group of $(U, G)$. Let $C=\mathbb{C}[X]^{M}$ and set

$$
C^{e}=\bigoplus_{\lambda \in \Gamma, m(\lambda)=e} \mathbb{C}[X]_{\lambda}^{M}, \quad C_{d}=\bigoplus_{e \leq d} C^{e} .
$$

Theorem 2. $C_{0} \subseteq C_{1} \subseteq \cdots \subseteq C_{j} \subseteq \cdots$ is a filtration of $C$.

Proof. It suffices to show: if $\lambda, \gamma \in G^{\wedge}, f \in U_{d} \subseteq \mathbb{C}[X], U_{d} \simeq \lambda, m(\lambda)=d$, $g \in U_{b} \subseteq \mathbb{C}[X], U_{b} \simeq \gamma, m(\gamma)=b, f, g \in \mathbb{C}[X]^{M}$, then $f \cdot g \in C_{b+d}$.

We have an epimorphism of $G$-modules $\gamma \otimes \lambda \rightarrow U_{b} \cdot U_{d}$. Let

$$
\gamma \otimes \lambda=\delta_{1} \oplus \cdots \oplus \delta_{t}, \quad \delta_{i} \in G^{\wedge} .
$$

Let us decompose $f \otimes g=h_{1}+\cdots+h_{s}, s \leq t, h_{i} \in \delta_{i}-0$, reordering the index set if necessary. We only need to check that $m\left(\delta_{i}\right) \leq b+d$, for all $i \leq s$. We have the following well-known isomorphism of $G$-modules:

$$
\mathbb{C}[G / M] \simeq \bigoplus_{\gamma \in G^{\wedge}} \gamma^{M} \otimes \gamma^{*}
$$

From the inclusions given by $f$ and $g \quad \lambda^{*} \hookrightarrow \lambda^{M} \otimes \lambda^{*}$ and $\gamma^{*} \hookrightarrow \gamma^{M} \otimes \gamma^{*}$ we have a morphism of $G$-modules

$$
\lambda^{*} \otimes \gamma^{*} \rightarrow\left(\lambda^{M} \otimes \lambda^{*}\right)\left(\gamma^{M} \otimes \gamma^{*}\right) \subseteq \bigoplus_{i=1, \ldots, t} \delta_{i}^{M} \otimes \delta_{i}^{*}
$$

Now we claim that $\left(\lambda^{M} \otimes \lambda^{*}\right)\left(\gamma^{M} \otimes \gamma^{*}\right) \supseteq \bigoplus_{i=1, \ldots, s} \delta_{i}^{M} \otimes \delta_{i}^{*}$. Indeed, if $c_{i} \in \delta_{i}^{*}$, there exist $\alpha_{j} \in \lambda^{*}, \beta_{j} \in \gamma^{*}$ such that $\sum c_{i}=\sum \alpha_{j} \otimes \beta_{j}$ in $\lambda^{*} \otimes \gamma^{*}$. Thus for every $x \in G$ :

$$
\begin{aligned}
\left\langle\sum x c_{i}, \sum h_{i}\right\rangle & =\left\langle\sum x \alpha_{j} \otimes x \beta_{j}, f \otimes g\right\rangle \\
& =\left\langle\sum x \alpha_{j}, f\right\rangle \cdot\left\langle\sum x \beta_{j}, g\right\rangle .
\end{aligned}
$$

That is, $\sum h_{i} \otimes c_{i}=\left(\sum f \otimes \alpha_{j}\right) \cdot\left(\sum g \otimes \beta_{j}\right)$.

Now, for any $v \in U$ such that $G v$ is closed and $G^{v}=M$, we have the following diagram of $G$-modules

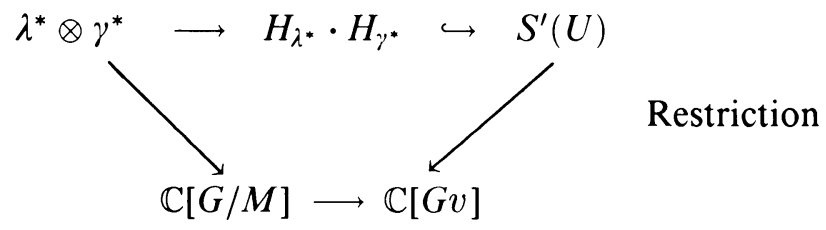

which is clearly commutative. Hence the homogeneous module, of degree $b+d$, $H_{\lambda^{*}} \cdot H_{\gamma^{*}}$ contains an irreducible $G$-module of type $\delta_{i}^{*}$ for each $i=1, \ldots, s$; as $S^{\prime}(U)_{\delta_{i}^{*}}=S^{\prime}(U)^{G} \cdot H_{\delta_{i}^{*}}$, we conclude that $m\left(\delta_{i}\right) \leq b+d$.

To generalize the preceding, we need the concept of a $\Delta$-filtration; it is surely well known, but as we do not know a good reference, we shall introduce it, along with some formal generalities. 
Let $(\Delta,+,<)$ be a monoid equipped with a partial order $<$, compatible with + , a p.o.m. for short. For example, let $A$ be a noetherian commutative $k$ algebra, $k$ a field; $\mathscr{S}(A)=\{W \subseteq A: W$ is a $k$-vector space $\}$ then $(\mathscr{S}(A), \cdot, \subseteq)$ is a p.o.m.

In such case, a $\Delta$-ascending filtration (resp., descending filtration) in $A$ is a morphism of p.o.m. sets $\Delta \stackrel{F}{\rightarrow} \mathscr{S}(A)$ (resp., $\Delta^{0} \stackrel{F}{\rightarrow} \mathscr{S}(A)$, where $\Delta^{0}$ is $\Delta$ with the order reversed) such that $F(a) F(b) \subseteq F(a+b)$ for all $a, b \in \Delta$ (resp., $F(a) F(b) \supseteq F(a+b))$ and if 0 is the identity of $\Delta$, then $k \subseteq F(0)$. Here we shall only consider the case of ascending filtrations.

So let $A$ be as above, equipped with a $\Delta$-filtration, and put $A_{d}=F(d)$, $d \in \Delta$. Clearly, $A_{0}$ is a $k$-subalgebra of $A$, and each $A_{d}$ is an $A_{0}$-module. Now put

$$
A_{(d)}=A_{d} /\left(\sum_{e<d} A_{e}\right) \text { and } \Delta-\operatorname{gr}(A)=\bigoplus_{d \in \Delta} A_{(d)} .
$$

The bilinear forms $A_{(d)} \times A_{(b)} \rightarrow A_{(d+b)}$ are well defined and extend to $\Delta-\operatorname{gr}(A)$, giving it a $\Delta$-graded $k$-algebra structure.

Examples. (i) Let $\Delta=\mathbb{N}_{0}^{s}, C=S^{\prime}(N)^{M}$ be as in Theorem 1. For $r \in \mathbb{N}_{0}^{s}$ put $C_{r}=\bigoplus_{\gamma \in \Gamma_{r}} S^{\prime}(W)_{\gamma}^{M}$. In fact, this gives a $\mathbb{N}_{0}^{s}$-filtration in $C$, as follows easily from Theorem 2.

Moreover, we have

Lemma 2. Let $D=\bigoplus_{r \in \mathbb{N}_{0}^{s}}\left(\bigoplus_{\gamma \in \Gamma, m(\gamma) \leq r} S^{\prime}(N)_{\gamma}^{M} \otimes S_{r}^{\prime}(A)\right)$ and let $J$ be the ideal of $D$ generated by $A^{*}$. Then

$$
D / J \simeq \Delta-\operatorname{gr}(C) .
$$

Proof. Left to the reader.

(ii) As in the classical case, if $A=\bigoplus_{d \in \Delta} A_{(d)}$ is a $\Delta$-graded $k$-algebra, then $A_{d}=\sum_{e \leq d} A_{(e)}$ induces a filtration in $A$; its $\Delta$-graded algebra is again $A$.

(iii) Let $\Delta=\mathbb{N}_{0}^{s}, t_{1}, \ldots, t_{N} \in \Delta$; we construct a $\Delta$-grading in the polynomial ring $B=k\left[X_{1}, \ldots, X_{N}\right]$ putting for $d \in \Delta$

$$
B_{(d)}=\left\langle\left\{X_{1}^{h_{1}} \cdots X_{N}^{h_{N}}: \sum_{i} h_{i} t_{i}=d\right\}\right\rangle .
$$

Now let $A$ be a noetherian commutative $k$-algebra equipped with a $\mathbb{N}_{0}^{s}$-filtration. The following result will be useful later.

Lemma 3. Let $x_{i} \in A_{t_{i}}(i=1, \ldots, N), \xi_{i}$ its image in $A_{\left(t_{i}\right)}$.

(A) If the $\xi_{i}$ are $k$-algebraically independent in $\Delta-\operatorname{gr}(A)$, then the $x_{i}$ are algebraically independent in $A$.

(B) If the $\xi_{i}$ generate $\Delta-\operatorname{gr}(A)$ as a k-algebra and $A=\bigcup_{d \in \mathbb{N}_{0}^{s}} A_{d}$, then the $x_{i}$ generate $A$.

Proof (As in [Bo, p. 39]). Consider $B \stackrel{\phi}{\rightarrow} A, X_{i} \mapsto x_{i}$. Clearly, $\phi\left(B_{d}\right) \subseteq A_{d}$ and hence we have $B \stackrel{\psi}{\rightarrow} \Delta-\operatorname{gr}(A)$.

(A) $\psi$ is $1-1$; thus $\psi: B_{d} / \sum_{e<d} B_{e} \rightarrow A_{d} / \sum_{e<d} A_{e}$ is $1-1$. Hence $\phi^{-1}(0) \subseteq$ $B_{0}$. But $\psi: B_{(0)} \rightarrow A_{(0)}$ is $\phi: B_{0} \rightarrow A_{0}$ thus $\phi$ is $1-1$.

(B) Left to the reader. 
Proposition 1. Let $(U, G)$ be a spherical representation, $N$ a finite-dimensional $G$-module, $\quad M$ a principal isotropy subgroup of $(U, G), A=U^{M}, W=$ $N_{G}(M) / M, C=S^{\prime}(N)^{M}$ with the filtration introduced above. Let

$$
D=\bigoplus_{n \in \mathbb{N}_{0}^{s}}\left(\bigoplus_{\gamma \in \Gamma, d(\gamma) \leq n} S^{\prime}(N)_{\gamma}^{M} \otimes S_{n}^{\prime}(A)\right)
$$

Consider the following statements:

(a) $S^{\prime}(U \oplus N)^{G}=D^{W}$ is a polynomial ring,

(b) $D$ is a polynomial ring,

(c) $\operatorname{gr} C$ is a polynomial ring,

(d) $S^{\prime}(N)^{M}$ is a polynomial ring;

Then $(\mathrm{a}) \Leftrightarrow(\mathrm{b}) \Leftrightarrow(\mathrm{c}) \Rightarrow(\mathrm{d})$

Proof. (b) $\Leftrightarrow$ (c): Let $H_{1}, \ldots, H_{s}$ be elements of a basis of $A^{*}$. As they form a regular sequence in $C \otimes S^{\prime}(A)$, they do in $D$. On the other hand, $D$ (and hence, $\operatorname{gr} C$ ) inherits the usual graded structure of $S^{\prime}(N) \otimes S^{\prime}(A)$. With respect to it, let $D_{+}$(resp., $(\mathrm{gr} C)_{+}$) be the maximal homogeneous ideal of $D$ (resp., of $\operatorname{gr} C)$. Thanks to Lemma 2 we have the following exact sequence

$$
0 \rightarrow\left\langle H_{1}, \ldots, H_{s}\right\rangle \rightarrow D_{+} /\left(D_{+}\right)^{2} \rightarrow(\operatorname{gr} C)_{+} /\left((\operatorname{gr} C)_{+}\right)^{2} \rightarrow 0 .
$$

Moreover, $D$ is regular iff Krull $\operatorname{dim} D=\operatorname{dim}_{\mathbb{C}} D_{+} /\left(D_{+}\right)^{2}$ (idem for $\operatorname{gr} C$ ). Let $J$ be the ideal of $D$ generated by $A^{*}$; as it is generated by a regular sequence, $\mathrm{ht}(J)=s$; since $D$ is an integral $\mathbb{C}$-algebra of finite type

$$
\text { Krull } \operatorname{dim}(\operatorname{gr} C)+\mathrm{ht}(J)=\operatorname{Krull} \operatorname{dim} D \text {. }
$$

But

$$
\begin{aligned}
\operatorname{Krull} \operatorname{dim}(\operatorname{gr} C)+\mathrm{ht}(J) & \leq \operatorname{dim}_{\mathbb{C}}(\operatorname{gr} C)_{+} /\left((\operatorname{gr} C)_{+}\right)^{2}+s \\
& =\operatorname{dim}_{\mathbb{C}} D_{+} /\left(D_{+}\right)^{2} \geq \operatorname{Krull} \operatorname{dim} D,
\end{aligned}
$$

and the announced equivalence follows easily.

(a) $\Leftrightarrow$ (b) From the proof of (b) $\Leftrightarrow$ (c) it follows that there exist $j(1), \ldots$, $j(s) \in \mathbb{N}_{0}^{s}, f_{i} \in E(N, M, j(i))$ for each $i$, such that

$$
D=\mathbb{C}\left[f_{i} H^{j(i)}, J_{1}, \ldots, H_{s}\right],
$$

where if $j \in \mathbb{N}_{0}^{s}, H^{j}=\prod_{k} H_{k}^{j(k)}$. But the $f_{i} H^{j(i)}$ are $W$-invariants (see the proof of Theorem 1); hence

$$
D^{W}=\mathbb{C}\left[f_{i} H^{j(i)}, H_{1}^{\nu(1)}, \ldots, H_{s}^{\nu(s)}\right],
$$

for some integers $\nu(i)$.

(c) $\Rightarrow$ (d) follows from Lemma 3 .

Remark 4. The implication (a) $\Rightarrow$ (d) is a particular case of the Luna Slice Etale Theorem application to Invariant Theory, see [KPV]. (b) $\Leftrightarrow(\mathrm{c})$ is a standard fact in commutative algebra; however it provides the interesting implication (c) $\Rightarrow$ (a). A word of caution: it does not follow from the proof of $(a) \Leftrightarrow(b)$ that $D$ is a polynomial ring over $\mathbb{C}\left[f_{i} H^{j(i)}\right]$; see [A2].

\section{THE CHARACTERIZATION OF THE ASSOCIATED GRADED RING}

Now let $(U, G)$ be a spherical representation, $\Delta=\mathbb{N}_{0}^{s}, \Delta-\operatorname{gr} S^{\prime}(N)^{M}=$ $\Delta$-gr $C$ as in example (i). We shall make the following hypothesis: 
(T) There exist a closed subgroup $P$ of $G$ and for each $\gamma \in \Gamma$, a "natural" isomorphism of vector spaces

$$
\gamma^{M} \stackrel{\phi}{\rightarrow} \gamma^{P}, \quad \phi=\phi(\gamma),
$$

satisfying: for each finite-dimensional $G$-module $N$, the bijective isomorphism

$$
\Delta-\operatorname{gr} C \rightarrow S^{\prime}(N)^{P},
$$

given by the $\phi(\gamma)$ is actually an isomorphism of $k$-algebras.

Now let $\left(U_{1}, G_{1}\right), \ldots,\left(U_{j}, G_{j}\right)$ be spherical representations and let $(U, G)$ $=\left(U_{1}, G_{1}\right) \oplus \cdots \oplus\left(U_{j}, G_{j}\right), M=M_{1} \times \cdots \times M_{j}, P=P_{1} \times \cdots \times P_{j}$, etc. Let us assume that $\left(U_{i}, G_{i}\right)$ satisfies (T) for $i=1, \ldots, j$. If $\gamma \in \Gamma, \gamma=\gamma_{1} \otimes \cdots \otimes \gamma_{j}$, we define

Then we have

$$
\gamma^{M} \stackrel{\phi}{\rightarrow} \gamma^{P}, \quad \phi=\phi(\gamma)=\phi\left(\gamma_{1}\right) \otimes \cdots \otimes \phi\left(\gamma_{j}\right) .
$$

Lemma 4. $(U, G), P,\{\phi(\gamma): \gamma \in \Gamma\}$ satisfies $(\mathrm{T})$.

Proof. It suffices to treat the case $j=2$. If $s_{i}$ is the rank of $\left(U_{i}, G_{i}\right), s=s_{1}+s_{2}$ is the rank of $(U, G)$. Thus if $t \in \mathbb{N}_{0}^{s}$, we shall denote $t=\left(t_{1}, t_{2}\right)$ in the obvious way. Let $N$ be a finite dimensional $G$-module. We introduce the following notation:

$$
E(N, M, t)=\bigoplus_{\gamma \in \Gamma, m(\gamma)=t} S^{\prime}(N)_{\gamma}^{M} .
$$

If $t=\left(t_{1}, t_{2}\right)$ observe that $E(N, M, t) \subseteq E\left(N, M_{i}, t_{i}\right)$ when we consider $N$ as $G_{i}$-module via the inclusion in the $i$-factor. Moreover $\gamma_{1}^{M_{1}} \otimes \gamma_{2}^{M_{2}} \rightarrow$ $\gamma_{1}^{P_{1}} \otimes \gamma_{2}^{P_{2}}$ is given by the composition ( $\left.\operatorname{Id} \otimes \phi\left(\gamma_{2}\right)\right) \circ\left(\phi\left(\gamma_{1}\right) \otimes \mathrm{Id}\right)$. Let us denote $\phi_{1}=\left(\phi\left(\gamma_{1}\right) \otimes\right.$ Id $)$, and similarly $\phi_{2}$.

Now let $f \in E(N, M, t), g \in E(N, M, r), f \cdot g=h_{0}+\cdots+h_{t+r}$ with $h_{l} \in E(N, M, l)$. Certainly, if $h_{l} \neq 0, l \leq t+r$. That is $l_{i} \leq t_{i}+r_{i}, i=1,2$. Hence $\phi_{1}(f) \phi_{1}(g)=\sum_{j: j_{1}=t_{1}+r_{1}} \phi_{1}\left(h_{j}\right)$, looking at $N$ as $G_{1}$-module.

Then $\phi_{1}(f) \in E\left(N, M_{2}, t_{2}\right), \phi_{1}(g) \in E\left(N, M_{2}, r_{2}\right)$, and

$$
\phi_{2}\left(\phi_{1}(f)\right) \cdot \phi_{2}\left(\phi_{1}(g)\right)=\phi_{2}\left(\phi_{1}\left(h_{t+r}\right)\right),
$$

i.e., $\phi(f) \cdot \phi(g)=\phi\left(h_{t+r}\right)$.

Now let $(U, G)$ be a spherical representation and let $G^{\prime} \stackrel{f}{\rightarrow} G$ be a finite covering. Then we may also consider the spherical representation $\left(U, G^{\prime}\right)$. Furthermore, if there exists a subgroup $P$ of $G$ satisfying the hypothesis (T), then the inverse image $P^{\prime}$ of $P$ in $G^{\prime}$ satisfies $(\mathrm{T})$ for $\left(U, G^{\prime}\right)$. (Alternatively, replace $N$ by the $G$-variety $N / \operatorname{Ker} f$; it corresponds to $S^{\prime}(N)^{\operatorname{Ker} f}=$ $\bigoplus_{\gamma \in G^{\wedge}} S^{\prime}(N)_{\gamma}$.)

We shall give a characterization, for $\gamma \in G^{\wedge}$, of $m(\gamma)$. We begin showing that every spherical representation of rank one is visible, i.e., the unstable cone has only a finite number of orbits, if $G$ is connected.

Theorem 3 [Se, 6.2]. If $G$ is a connected reductive linear group and $Y$ is an irreducible affine $G$-variety such that $\mathbb{C}[Y]$ contains each $\gamma \in G^{\wedge}$ at most once, then $G$ has only a finite number of orbits in $Y$.

Lemma 5. Let $(U, L)$ be an irreducible representation of a connected algebraic reductive group $L$ such that $S^{\prime}(U)^{L}$ is a polynomial ring generated by a single 
invariant, say $J$. Let $\pi: U \rightarrow U / L$ be the application associated to the inclusion, let $\mathfrak{N}=\mathfrak{N}(U, L)=\pi^{-1}(\pi(0))$ be the unstable cone. Then $\mathfrak{N}$ is irreducible and the ideal associated to $\mathfrak{N}$ is $S^{\prime}(U) J$.

Proof. Note that $\mathfrak{N}$ is the zero set of $J$. If $L$ is semisimple, a standard argument shows that $J$ is irreducible in $S^{\prime}(U)$ : let $J=p_{1} \cdots p_{s}$ be the factorization of $J$ in primes; then for each $i$ there exists a character $\chi_{i}$ of $L$ such that for every $k \in L, k \cdot p_{i}=\chi_{i}(k) p_{i}$, but $L$ has no nontrivial characters. But in our case, as $(U, L)$ is irreducible, the "nonsemisimple" part of $L$ acts on $U$ by a single character $\chi$; hence $\chi_{i}=\chi^{\operatorname{deg} p_{i}}$. It follows that the image of $\chi$ is finite, but $L$ is connected.

Proposition 2. Let $(U, G)$ be a spherical representation of rank one, $G$ connected. Then $\mathfrak{N}(U, G)$ contains only a finite number of orbits. Moreover, for each $x \in \mathfrak{N},\left(G, G^{x}\right)$ is a spherical pair.

Proof. We want to apply the above-quoted theorem of Servedio to $Y=\mathfrak{N}$. We only need to observe that the restriction $S^{\prime}(U) \rightarrow \mathbb{C}[\mathfrak{N}]$ induces an epimorphism $H \rightarrow \mathbb{C}[\mathfrak{N}]$. (Recall the decomposition $S^{\prime}(U)=S^{\prime}(U)^{G} \otimes H$.) The second assertion follows from [VK, Corollary 3].

Remark 5. The last result reduces drastically the list of candidates of possible spherical representations; see [Kc]. We now generalize an argument of Kostant:

Let $(U, L)$ be a cofree representation of an algebraic reductive group $L$, $\pi$ as in Lemma 5. Let $H$ be a homogeneous subspace of $S^{\prime}(U)$ such that $S^{\prime}(U)=S^{\prime}(U)^{L} \otimes H$. We set $F(\gamma)=\operatorname{Hom}_{L}(\gamma, H)$, for $\gamma \in L^{\wedge}$ and we fix $\gamma$ such that $F\left(\gamma^{*}\right) \neq 0$. Let $d_{1}, \ldots, d_{m}$ be the degrees of homogeneity of $\gamma^{*}$ in $H ; m=\operatorname{dim} F\left(\gamma^{*}\right)$. We shall assume that the ideal $S^{\prime}(U)_{+}^{L} \cdot S^{\prime}(U)$ is prime and that there exists an $x \in \mathfrak{N}(U, L)$ such that the closure of the orbit $L \cdot x$ is $\mathfrak{N}$. Now let $P=L^{x}, P^{\prime}=$ normalizer of $\mathbb{C} x$ in $L ; P^{\prime}$ acts in $\mathbb{C} x$ via a character $\chi$. Finally, for any $y \in U, \sigma \in F\left(\gamma^{*}\right), \alpha \in \gamma^{*}$, let $\beta_{y}: F\left(\gamma^{*}\right) \rightarrow \gamma^{G^{y}}$ be given by

$$
\left\langle\beta_{y}(\sigma), \alpha\right\rangle=\sigma(\alpha)(y) .
$$

Proposition 3. $\beta_{x}$ is one-to-one; moreover, $P^{\prime}$ acts in $\operatorname{Im} \beta_{x}$ decomposing it in $P^{\prime}$-submodules of dimension one and the associated characters are precisely of the form $\chi^{d_{1}}, \ldots, \chi^{d_{m}}$. Finally, the $d_{i}$ 's are determined by this fact.

Proof. The injectivity of $\beta_{x}$ and the following identity, from which the second assertion is deduced, can be found for example in $[\mathrm{K}]$ :

$$
\text { for all } a \in G: \quad a \cdot \beta_{x}(\sigma)=\beta_{a \cdot x}(\sigma) .
$$

Now $L \cdot x$ is open in $\mathfrak{N}$ and $\mathbb{C}_{x} \subseteq \mathfrak{N}$; thus $P^{\prime} \cdot x=L \cdot x \cap \mathbb{C} x$ is a nonempty open subset of $\mathbb{C} x$ and hence the image of $\chi$ cannot be finite.

If $(U, G)$ is a spherical representation of rank one and $x \in \mathfrak{N}(U, G)$ is such that $L x$ is dense in $\mathfrak{N}, \beta_{x}$ is an isomorphism of vector spaces for all $\gamma \in \Gamma$, because $(G, P)$ is a spherical pair. Clearly $\left\{\gamma \in \Gamma: \gamma^{P} \neq 0\right\} \supseteq \Gamma$; so let us assume that the equality holds. This is true if for example, the codimension in $\mathfrak{N}$ of $(\mathfrak{N}-G x)$ is $\geq 2$ (see $[\mathrm{K}]: \mathfrak{N}$ is normal since it is an irreducible hypersurface). For those spherical representations of simple groups, one can check that the condition is fulfilled in view of [Po3]. On the other hand, $\beta_{y}$ is 
also an isomorphism for every $y \in U^{M}-0$. Fix such a $y$. We define $\phi=\phi(\gamma)$ by making commutative the following diagram:

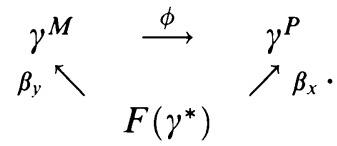

Remark 6. Let us assume that the codimension in $\mathfrak{N}$ of $(\mathfrak{N}-G x)$ is $\geq 2$. Then the equality $\left\{\rho \in G^{\wedge}: \rho^{M} \neq 0\right\}=\left\{\rho \in G^{\wedge}: \rho^{P} \neq 0\right\}$ can be viewed as a generalization of the well-known Cartan-Helgason Theorem. Indeed, when $\mathfrak{g}_{\mathbb{R}}=\mathfrak{s o}(n, 1)$ the claimed equality is a consequence of Cartan-Helgason.

Theorem 4. The isomorphisms $\{\phi(\gamma): \gamma \in \Gamma\}$ give rise to an isomorphism of algebras between $\operatorname{gr}\left(\mathbb{C}[X]^{M}\right)$ and $\mathbb{C}[X]^{P}$, for every $G$-variety $X$.

Proof. We shall denote by $h^{\sharp}$ the image of $h \in \tau^{M}$ by $\phi$. Let $f \in \gamma^{M}$, $g \in \lambda^{M}$. We have

$$
\gamma \otimes \lambda=\delta_{1} \oplus \cdots \oplus \delta_{t}, \quad \delta_{i} \in G^{\wedge} .
$$

Let us decompose $f \otimes g=h_{1}+\cdots+h_{r}+\cdots+h_{s}, r \leq s \leq t, h_{i} \in \delta_{i}-0$. Reordering the index set if necessary, we may assume $m\left(\delta_{i}\right)=m(\gamma)+m(\lambda)$ if $i \leq r$ and $m\left(\delta_{i}\right)<m(\gamma)+m(\lambda)$ if $r<i \leq s$. We only need to check that $f^{\sharp} \otimes g^{\sharp}=h_{1}^{\sharp}+\cdots+h_{r}^{\sharp}$. Let $\mathscr{A} \in F\left(\gamma^{*}\right), \mathscr{B} \in F\left(\lambda^{*}\right), \mathscr{C}_{i} \in F\left(\delta_{i}^{*}\right)$ corresponding to $f, g, h_{i}$. This means, for example, that if $u \in \gamma^{*}$

$$
\langle f, u\rangle=\mathscr{A}(u)(y), \quad\left\langle f^{\sharp}, u\right\rangle=\mathscr{A}(u)(x) .
$$

So let $u \in \gamma^{*}, v \in \lambda^{*}$; with the above identification, there exist $w_{i} \in \delta_{i}^{*}$ such that

$$
u \otimes v=w_{1}+\cdots+w_{t}
$$

Hence

$$
\langle f \otimes g, u \otimes v\rangle=\left\langle h_{1}, w_{1}\right\rangle+\cdots+\left\langle h_{r}, w_{r}\right\rangle+\cdots+\left\langle h_{s}, w_{s}\right\rangle
$$

i.e.,

$$
\mathscr{A}(u)(y) \mathscr{B}(v)(v)=\mathscr{C}_{1}\left(w_{1}\right)(y)+\cdots+\mathscr{C}_{r}\left(w_{r}\right)(y)+\cdots+\mathscr{C}_{s}\left(w_{s}\right)(y) .
$$

Let us denote by $J$ a homogeneous generator of $S^{\prime}(U)^{G}$. As $\mathscr{C}_{i}\left(w_{i}\right) \in H$ for all $i$, there exist integers $d_{r+1}, \ldots, d_{s}$ such that $J^{d_{i}} \mathscr{C}_{i}\left(w_{i}\right)$ is homogeneous of degree $m(\gamma)+m(\lambda)$. Put $j_{i}=\left(J^{d_{i}}(y)\right)^{-1}$. Hence $\mathscr{A}(u) \mathscr{B}(v)$ and

$$
\mathscr{C}_{1}\left(w_{1}\right)+\cdots+\mathscr{C}_{r}\left(w_{r}\right)+j_{r+1} J^{d_{r+1}} \mathscr{C}_{r+1}\left(w_{r+1}\right) \cdots+j_{s} J^{d_{s}} \mathscr{C}_{s}\left(w_{s}\right)
$$

are homogeneous polynomials which agree on $y$, hence on $k \cdot y$ for all $k \in G$; if they agree on $z$, they do on $t z$ for all $t \in \mathbb{C}^{\times}$. It follows that they agree on the whole of $U$. In particular, as $x \in \mathfrak{N}=\{z \in U: J(z)=0\}$, we have

$$
\mathscr{A}(u)(x) \mathscr{B}(v)(x)=\mathscr{C}_{1}\left(w_{1}\right)(x)+\cdots+\mathscr{C}_{r}\left(w_{r}\right)(x),
$$

i.e.,

$$
\left\langle f^{\sharp} \otimes g^{\sharp}, u \otimes v\right\rangle=\left\langle h_{1}^{\sharp}, w_{1}\right\rangle+\cdots+\left\langle h_{r}^{\sharp}, w_{r}\right\rangle .
$$

Thanks to Lemma 4 , Theorem 4 generalizes to spherical representations of arbitrary rank, provided that the hypothesis discussed above is fulfilled for each factor of $G$. Theorem 4 combined with Proposition 1 gives: 
Theorem 5. Let $(U, G)$ be a spherical representation, $N$ a finite-dimensional $G$-module, $x \in \mathfrak{N}(U, G)$ such that the closure of the orbit $G \cdot x$ is $\mathfrak{N}$ and the codimension in $\mathfrak{N}$ of $(\mathfrak{N}-G x)$ is $\geq 2$. Now let $P=G^{x}$. Then $S^{\prime}(U \oplus N)^{G}$ is a polynomial ring if and only if $S^{\prime}(N)^{P}$ is a polynomial ring.

\section{SOME EXAMPLES}

Let us retain the notation of the introduction. As a first application, we have:

Theorem 6. $S^{\prime}(\mathfrak{g})^{K}$ is a polynomial ring if $\mathfrak{g}_{\mathbb{R}}=\mathfrak{s o}(n, 1)$ or $\mathfrak{s u}(n, 1)$.

Proof. We observe that Theorems $1,2,4$, and 5 apply if $(U, G)$ is $(\mathfrak{p}, K)$ and $A$ is a one-dimensional Cartan subspace, even if $A \neq \mathfrak{p}^{M}$ with the following slight modification:

$$
\operatorname{gr}\left(\mathbb{C}[X]^{M}\right) \simeq \mathbb{C}[X]_{\Gamma}^{P}=\oplus_{\gamma \in \Gamma} \mathbb{C}[X]_{\gamma}^{P} .
$$

Now if $\mathfrak{g}_{\mathbb{R}}=\mathfrak{s o}(n, 1)$ then we may assume that $K=S O(n, \mathbb{C})$; it is known that $\mathfrak{p}$ is the natural representation. Let $E \in \mathfrak{p}$ be a highest weight vector, with respect to a fixed Borel subalgebra $\mathfrak{b}=\mathfrak{h} \oplus \mathfrak{n}$ of $\mathfrak{k}$. We claim that $E \in \mathfrak{N}(\mathfrak{p}, K)$ and that $K E$ has maximal dimension. The first is clear: $0=\lim _{t \rightarrow 0, t \in \mathbb{C}^{*}} \Lambda(t) E$, where $\psi$ is the highest weight of $\mathfrak{p}$ and $\Lambda$ is the one parameter subgroup dual to $\psi$. It is known that $\operatorname{dim} \mathfrak{N}(\mathfrak{p}, K)=\operatorname{dim} \mathfrak{p}-\operatorname{dim} \mathfrak{p} / K=\operatorname{dim} \mathfrak{p}-1$ so for the second it suffices to prove that $\operatorname{dim} K E=\operatorname{dim} \mathfrak{p}-1$, that is

$$
\operatorname{dim} K-\operatorname{dim} K^{E}=\operatorname{dim} \mathfrak{p}-1,
$$

which is equivalent in our case to

$$
\operatorname{dim} K^{E}=\left(n^{2}-n\right) / 2-n+1,
$$

or even to $\operatorname{dim} \mathfrak{k}^{E}=\left(n^{2}-n\right) / 2-n+1$, which is very easy to verify. So let us put $P=K^{E}$. Clearly $P \supseteq N$ where $N$ is the maximal unipotent subgroup corresponding to $\mathfrak{n}$. Let us denote by $V(\tau)$ the irreducible $\mathfrak{k}$-module of highest weight $\tau \in \mathfrak{h}^{*}$. We claim that

$$
\Gamma=\left\{\gamma \in K^{\wedge}: \gamma=V(j \psi), j \in \mathbb{N}_{0}\right\},
$$

and that if $\gamma \in \Gamma$ then $\gamma^{P}=\gamma^{N}$. This second statement is clearly true. Let $\Psi$ be the character of $\operatorname{Ad} \mathfrak{h}$ corresponding to $\psi$, i.e., $\Psi=\exp \psi$. Clearly $P \supseteq \operatorname{Ker} \Psi$; if $f \in S^{\prime}(\mathfrak{p})_{\psi}^{N}$ then $f^{j} \in S^{\prime}(\mathfrak{p})_{j \psi}^{N}$ and hence $\Gamma \supseteq\left\{\gamma \in K^{\wedge}: \gamma=\right.$ $\left.V(j \psi), j \in \mathbb{N}_{0}\right\}$. But if $\gamma \in \Gamma$ has highest weight $\xi$ and $\Xi=\exp \xi$, then $\gamma^{N}$ is stabilized by $\operatorname{Ker} \Xi \cdot \operatorname{Ker} \Psi$ and the other inclusion follows. Thus

$$
S^{\prime}(\mathfrak{k})^{P}=\bigoplus_{\gamma \in \Gamma} S^{\prime}(\mathfrak{k})_{\gamma}^{N}=\bigoplus_{j \geq 0} S^{\prime}(\mathfrak{k})_{j \psi}^{N}=\bigoplus_{j \geq 0} S^{\prime}(\mathfrak{k})_{2 j \psi}^{N},
$$

because we know from $[\mathrm{K}]$ that an irreducible representation arises in the coordinate ring of the adjoint representation if and only if its highest weight lives in the root lattice. Now a theorem of Levstein (see Theorem 7 below) guarantees that $S^{\prime}(\mathfrak{k})^{P}$ is a polynomial ring; we conclude from Theorem 5 that $S^{\prime}(\mathfrak{g})^{K}$ is a polynomial ring too.

On the other hand, if $\mathfrak{g}_{\mathbb{R}}=\mathfrak{s u}(n, 1)$ then $\mathfrak{k}=\mathfrak{s l}(n, \mathbb{C}) \oplus \mathbb{C}$ and it is well known that $\mathfrak{p}=\mathfrak{p}_{-} \oplus \mathfrak{p}_{+}$where $\mathfrak{s l}(n, \mathbb{C})$ (resp., $\mathbb{C}$ ) acts in $\mathfrak{p}_{+}$via the natural 
representation (respectively, via a nontrivial character) and $\mathfrak{p}_{-}$is the dual of $\mathfrak{p}_{+}$. In fact, one can choose a realization as follows: $\mathfrak{g}=\mathfrak{s l}(n+1, \mathbb{C})$,

$$
\begin{aligned}
& \mathfrak{k}=\left\{\left(\begin{array}{ll}
A & 0 \\
0 & a
\end{array}\right): A \in \mathfrak{g l}(n, \mathbb{C}), \operatorname{tr} A+a=0\right\}, \\
& \mathfrak{p}=\left\{\left(\begin{array}{ll}
0 & u \\
v & 0
\end{array}\right): u \in \mathbb{C}^{n \times 1}, v \in \mathbb{C}^{1 \times n}\right\} .
\end{aligned}
$$

Let $\mathfrak{b}=\mathfrak{h} \oplus \mathfrak{n}$ be the Borel subalgebra of $\mathfrak{k}$ of upper triangular matrices in $\mathfrak{k}$, where $\mathfrak{h}$ are the diagonal matrices in $\mathfrak{k}$, and let $E_{+} \in \mathfrak{p}_{+}$(resp., $E_{-} \in \mathfrak{p}_{-}$) be given by $u=e_{1}, v=0$ (resp., $u=0, v=e_{n}$ ). We claim that $E=E_{+}+E_{-} \in$ $\mathfrak{N}(\mathfrak{p}, K)$ and that $K E$ has maximal dimension. The first statement is easy and the second will follow from

$$
\operatorname{dim} K^{E}=n^{2}-2 n+1,
$$

or even from

$$
\operatorname{dim} \mathfrak{k}^{E}=n^{2}-2 n+1 \text {. }
$$

But

$$
\begin{aligned}
\mathfrak{k}^{E} & =\left\{Z \in \mathfrak{k}: Z E_{+}+Z E_{-}=0\right\} \\
& =\left\{Z=Z_{1}+Z_{2} \in \mathfrak{k}: Z_{1} E_{+}+Z_{2} E_{+}=Z_{1} E_{-}-Z_{2} E_{-}=0\right\} \\
& =\left\{\left(\begin{array}{cccc}
z & { }^{t} u & w & 0 \\
0 & A & v & 0 \\
0 & 0 & z & 0 \\
0 & 0 & 0 & z
\end{array}\right) \in \mathfrak{k}: A \in \mathfrak{g l}(n-2, \mathbb{C}), z \in \mathbb{C}\right\},
\end{aligned}
$$

which has the necessary dimension. Thus

$$
S^{\prime}(\mathfrak{k})^{P}=S^{\prime}([\mathfrak{k}, \mathfrak{k}])_{\Gamma^{\prime}}^{P} \otimes S^{\prime}(\mathfrak{c}),
$$

where $\mathfrak{c}$ is the one-dimensional center and $\Gamma^{\prime}=\left\{\gamma \in \operatorname{PSL}(n, \mathbb{C})^{\wedge}: \gamma \otimes \mathrm{id} \in \Gamma\right\}$. Let $\psi$ (resp., $\psi^{*}$ ) be the dominant weight of the natural representation (resp., of its dual). We claim that

$$
\Gamma^{\prime}=\left\{V\left(j\left(\psi+\psi^{*}\right)\right), j \in \mathbb{N}_{0}\right\},
$$

and that if $\gamma \in \Gamma^{\prime}$ then $\gamma^{P}=\gamma^{N}$. In fact, the second assertion is easy and we can show that $V\left(j\left(\psi+\psi^{*}\right)\right) \in \Gamma^{\prime}$ by induction on $j$. The other inclusion follows as above; again, Levstein's result and Theorem 5 guarantee that $S^{\prime}(\mathfrak{g})^{K}$ is a polynomial ring.

Theorem 7. Let $\mathfrak{l}$ be a classical simple complex Lie algebra, $\mathfrak{b}$ a Borel subalgebra and let $\psi$ (resp., $\psi^{*}$ ) be the highest weight (with respect to $\mathfrak{b}$ ) of the natural representation of $\mathfrak{l}$ (resp., of its dual). Let $\mathfrak{u}=[\mathfrak{b}, \mathfrak{b}] \oplus \operatorname{Ker}\left(\psi+\psi^{*}\right)$. Then $S^{\prime}(\mathfrak{l})^{\mathfrak{u}}$ is a polynomial ring.

Proof. See [L].

Remark 7. It was shown in [A3] that these are the only cases for which $S^{\prime}(\mathfrak{g})^{K}$ is regular. Moreover, Theorem 6 was proved in [C] by geometric considerations; and the coregularity of $(\mathfrak{g}, K)$ when $\mathfrak{g}_{\mathbb{R}}=\mathfrak{s o}(n, 1)$ (resp., $\left.\mathfrak{s} \mathfrak{u}(n, 1)\right)$ is also proved in $[\mathrm{AG}, \mathrm{B}, \mathrm{Sch}]$ (resp., $[\mathrm{J}]$ ).

In order to get a second application let us recall the following: 
Theorem 8. $S^{\prime}(\mathfrak{p})^{P}$ is a polynomial ring if $\mathfrak{g}_{\mathbb{R}}$ is classical of rank one.

Proof. See [BT, Theorem 3.14].

Theorem 5 says that this is equivalent to

Theorem 9. $S^{\prime}(\mathfrak{p} \oplus \mathfrak{p})^{K}$ is a polynomial ring if $\mathfrak{g}_{\mathbb{R}}$ is classical of rank one.

Remark 8. Theorem 9 follows from classical invariant theory if $\mathfrak{g}_{\mathbb{R}}=\mathfrak{s o}(n, 1)$ or $\mathfrak{s u}(n, 1)$; so in this case Theorem 8 can be deduced from Theorem 5 . On the other hand, Theorem 9 seems to be new if $\mathfrak{g}_{\mathbb{R}}=\mathfrak{s p}(n, 1)$. More explicitly, let $V_{n}$ be the natural representation of $\mathfrak{s p}(n, \mathbb{C})$; then

$$
S^{\prime}\left(\left(V_{n} \otimes V_{1}\right) \oplus\left(V_{n} \otimes V_{1}\right)\right)^{\mathrm{SP}(n, \mathbb{C}) \times \mathrm{SP}(1, \mathbb{C})}
$$

is regular.

We conclude this section by giving an explicit presentation of the ring $S^{\prime}(\mathfrak{g})^{K}$ when $\mathfrak{g}_{\mathbb{R}}=\mathfrak{s p}(2,1)$. The case $\mathfrak{s p}(1,1) \simeq \mathfrak{s o}(4,1)$ is covered by Theorem 6 ; it turns out (at least as far as we know) that $\mathfrak{s p}(2,1)$ is the first nonregular $(\mathfrak{g}, K)$ of rank one computed in the literature. The strategy is as follows: first we compute $S^{\prime}(\mathfrak{k})^{P}$, where $P$ is the isotropy subgroup of a nilpotent element in $\mathfrak{p}$ whose orbit has maximal dimension. It turns out that it is a hypersurface; from Theorem 4 we can conclude that $S^{\prime}(\mathfrak{k})^{M}$ is also a hypersurface. Then we compute the generators and the relation of $S^{\prime}(\mathfrak{k})^{M}$. Using this information and Theorem 1, we give the generators and relation of the image by the restriction morphism of $S^{\prime}(\mathfrak{g})^{K}$. The details of these last two computations were presented in [A2].

Let us fix some notation. We have

$$
\begin{gathered}
\mathfrak{g}=\mathfrak{s p}(3, \mathbb{C}) \\
=\left\{\left(\begin{array}{ll}
Z_{1} & Z_{2} \\
Z_{3} & Z_{4}
\end{array}\right)=Z: Z_{i} \in \mathbb{C}^{3 \times 3}, Z_{1}=-{ }^{t} Z_{4}, Z_{2}, Z_{3} \text { symmetric }\right\}, \\
\mathfrak{k}=\left\{Z \in \mathfrak{g}: Z_{1}=\left(\begin{array}{ll}
A & 0 \\
0 & \alpha
\end{array}\right), Z_{2}=\left(\begin{array}{ll}
B & 0 \\
0 & \beta
\end{array}\right), Z_{3}=\left(\begin{array}{ll}
C & 0 \\
0 & \gamma
\end{array}\right), A, B, C \in \mathbb{C}^{2 \times 2}, \alpha, \beta, \gamma \in\right. \\
\mathbb{C}\} \simeq \mathfrak{k}_{1} \times \mathfrak{k}_{2} \text { where } \mathfrak{k}_{1}=\mathfrak{s p}(2, \mathbb{C}), \mathfrak{k}_{2}=\mathfrak{s l}(2, \mathbb{C}) \text {. We denote the entries of } \\
A, B, C \text { as } a_{1}, \ldots, b_{1}, b_{2}, b_{3}, c_{1}, \text { etc. where }
\end{gathered}
$$

We will think of the $a_{i}, b_{j}$, etc. as elements of $\left(\mathfrak{k}_{1}\right)^{*}$. We have

$$
\begin{aligned}
K=\{ & \left(\left(\begin{array}{ll}
T & Q \\
Z & V
\end{array}\right),\left(\begin{array}{cc}
t & y \\
v & z
\end{array}\right)\right): T, Q, V, Z \in \mathbb{C}^{2 \times 2} \cdot t, y, v, z \in \mathbb{C} \\
& \text { s.t. } \left.{ }^{t} T V-{ }^{t} Z Q=I,{ }^{t} T Z-{ }^{t} Z T=0={ }^{t} Q V-{ }^{t} V Q, t z-y v=1\right\} .
\end{aligned}
$$

As a $\mathfrak{k}_{1} \times \mathfrak{k}_{2}$-module, $\mathfrak{p}$ is then isomorphic to $\lambda_{1}\left(\mathfrak{k}_{1}\right) \otimes \lambda_{1}\left(\mathfrak{k}_{2}\right)$, where $\lambda_{1}$ means in each case the natural representation on $\mathbb{C}^{4}$ or $\mathbb{C}^{2}$. (This is in general true for $\mathfrak{s p}(n, 1)$, where $\mathfrak{k}_{1}=\mathfrak{s p}(n, \mathbb{C})$, etc. $)$. We shall denote $\mathfrak{p}=\left\{\left(\begin{array}{ll}u & x \\ r & u\end{array}\right)\right\}=\mathbb{C}^{4 \times 2}$, where the action is given by

$$
(x, y) \cdot P=x P-P \mathscr{y} \quad\left(x \in \mathfrak{k}_{1}, y \in \mathfrak{k}_{2}, P \in \mathfrak{p}\right) .
$$


Furthermore, if $X \in \mathfrak{p}$ is given by $u=w=e_{1}, r=x=0$, then we can choose $\mathfrak{a}=\mathbb{C} \cdot X$ and hence $M$ is the connected subgroup of $K$ corresponding to

$$
\mathfrak{m}=\left\{\left(\left(\begin{array}{cccc}
\alpha & 0 & \beta & 0 \\
0 & a & 0 & b \\
\gamma & 0 & -\alpha & 0 \\
0 & c & 0 & -a
\end{array}\right),\left(\begin{array}{cc}
\alpha & \beta \\
\gamma & -\alpha
\end{array}\right)\right) \in \mathfrak{k}: \alpha, \beta, \gamma, a, b, c \in \mathbb{C}\right\},
$$

$\mathfrak{m}=\mathfrak{m}_{1} \times \mathfrak{m}_{2}$, where $m_{i} \simeq \mathfrak{s l}(2, \mathbb{C}), i=1,2$, in an evident way.

As an $M$-module, $\mathfrak{k}_{1}$ is isomorphic to $\mathrm{Ad}_{1}+\mathrm{Ad}_{2}+\mathfrak{p}^{\sim}$, where

$$
\mathfrak{p}^{\sim}=\left\{X \in \mathfrak{k}_{1}: a_{1}=a_{4}=b_{1}=b_{3}=c_{1}=c_{3}=0\right\} .
$$

Remark 9. $\left(\mathfrak{k}_{1}, M\right)=(\mathfrak{g}, K)$ for $\mathfrak{s p}(1,1) \simeq \mathfrak{s o}(4,1)$; as we noted above, $S^{\prime}(\mathfrak{g})^{K}$ is a polynomial ring in 4 variables.

We shall also fix a Cartan subalgebra $\mathfrak{t}=\mathfrak{t}_{1} \times \mathfrak{t}_{2} \subseteq \mathfrak{k}, \mathfrak{t}_{i} \subseteq \mathfrak{k}_{i}$ are the diagonal matrices.

Finally, let $Y \in \mathfrak{p}$ be given by $u=e_{1}, x=e_{2}, w=r=0$. Let

$$
\gamma_{t}=\left(\left(\begin{array}{cc}
T & 0 \\
0 & T^{-1}
\end{array}\right),\left(\begin{array}{ll}
1 & 0 \\
0 & 1
\end{array}\right)\right) \in K
$$

with $T=t I, t \in \mathbb{C}$. Then $\gamma_{t} \cdot Y$ is given by $u=t e_{1}, x=t e_{2}, w=r=0$ and this shows that $Y$ is nilpotent.

The isotropy subgroup of $Y$ in $K$ is

$$
\begin{array}{r}
P=\left\{\left(\left(\begin{array}{ll}
T & Q \\
0 & V
\end{array}\right),\left(\begin{array}{ll}
t & y \\
v & z
\end{array}\right)\right): T=\left(\begin{array}{ll}
t & y \\
v & z
\end{array}\right), Q=\left(\begin{array}{ll}
e & f \\
g & h
\end{array}\right),\right. \\
\left.V=\left(\begin{array}{cc}
z & -v \\
-y & t
\end{array}\right), t g-v e=z f-y h, t z-y v=1\right\} .
\end{array}
$$

As $\operatorname{dim} K Y=\operatorname{dim} K-\operatorname{dim} P$ is maximal, we conclude that $Y$ is principal nilpotent. It is easy to see that $P=R H$, where

$$
\begin{aligned}
R=\left\{\left(\left(\begin{array}{ll}
T & 0 \\
0 & V
\end{array}\right),\left(\begin{array}{cc}
t & y \\
v & z
\end{array}\right)\right): T=\left(\begin{array}{cc}
t & y \\
v & z
\end{array}\right),\right. \\
\left.V=\left(\begin{array}{cc}
z & -v \\
-y & t
\end{array}\right), t z-y v=1\right\},
\end{aligned}
$$

and

$$
H=\left\{\left(\left(\begin{array}{ll}
I & Q \\
0 & I
\end{array}\right),\left(\begin{array}{ll}
1 & 0 \\
0 & 1
\end{array}\right)\right): Q=\left(\begin{array}{ll}
e & f \\
f & h
\end{array}\right)\right\} .
$$

In other words, $H$ is the (abelian, three-dimensional) unipotent radical of $P$ and $R$ (a copy of $\mathrm{SL}(2, \mathbb{C})$ ) is a Levi factor.

Our first step is to compute $S^{\prime}(\mathfrak{k})^{H} \simeq S^{\prime}\left(\mathfrak{k}_{1}\right)^{H} \otimes S^{\prime}\left(\mathfrak{t}_{2}\right)$. The action of $H$ in $\mathfrak{k}_{1}$ is of course given by

$$
\left(\begin{array}{ll}
I & Q \\
0 & I
\end{array}\right)\left(\begin{array}{cc}
A & B \\
C & -{ }^{t} A
\end{array}\right)\left(\begin{array}{cc}
I & -Q \\
0 & I
\end{array}\right)=\left(\begin{array}{lr}
A+Q C & B-Q^{t} A-A Q-Q C Q \\
C & -C Q-{ }^{t} A
\end{array}\right) .
$$


Thus $\left(\begin{array}{cc}I & -Q \\ 0 & I\end{array}\right)$ acts as follows in $S^{\prime}\left(\mathfrak{k}_{1}\right)$ (if $\left.Q=\left(\begin{array}{ll}e & f \\ f & h\end{array}\right)\right)$ :

$$
\begin{aligned}
a_{1} & \mapsto a_{1}+e c_{1}+f c_{2}, \\
a_{2} & \mapsto a_{2}+e c_{2}+f c_{3}, \\
a_{3} & \mapsto a_{3}+f c_{1}+h c_{2}, \\
a_{4} & \mapsto a_{4}+f c_{2}+h c_{3}, \\
c_{i} & \mapsto c_{i}, i=1,2,3, \\
b_{1} & \mapsto b_{1}-2\left(e a_{1}+f a_{2}\right)-\left(e^{2} c_{1}+2 e f c_{2}+f^{2} c_{3}\right), \\
b_{2} & \mapsto b_{2}-\left(f a_{1}+h a_{2}+e a_{3}+f a_{4}\right)-\left(e f c_{1}+\left(f^{2}+e h\right) c_{2}+f h c_{3}\right), \\
b_{3} & \mapsto b_{3}-2\left(f a_{3}+h a_{4}\right)-\left(f^{2} c_{1}+2 f h c_{2}+h^{2} c_{3}\right) .
\end{aligned}
$$

Let us introduce the following polynomials in $\mathfrak{k}_{1}$ :

$$
\begin{aligned}
\Delta= & c_{2}^{2}-c_{1} c_{3}, \\
\vartheta_{1}= & a_{4} c_{2}+a_{2} c_{1}-a_{1} c_{2}-a_{3} c_{3}, \\
\vartheta_{2}= & b_{1} \Delta-a_{1}^{2} c_{3}+2 a_{1} a_{2} c_{2}-a_{2}^{2} c_{1}, \\
\vartheta_{3}= & \Delta\left(b_{2} c_{2}+a_{2} a_{3}\right)-\left(a_{2} c_{1}-a_{1} c_{2}\right)\left(a_{4} c_{2}-a_{3} c_{3}\right), \\
\vartheta_{4}= & \Delta\left(b_{3} c_{2}^{2}-a_{3}^{2} c_{3}+2 a_{3} a_{4} c_{2}\right) \\
& +c_{1}\left[\left(a_{2} c_{1}-a_{1} c_{2}\right)^{2}+2\left(a_{2} c_{1}-a_{1} c_{2}\right)\left(a_{4} c_{2}-a_{3} c_{3}\right)\right], \\
\vartheta_{5}= & \Delta\left(b_{2} c_{1} c_{3}-a_{1} a_{4} c_{2}+a_{2} a_{4} c_{1}+a_{1} a_{3} c_{3}\right) \\
& -c_{2}\left(a_{2} c_{1}-a_{1} c_{2}\right)\left(a_{4} c_{2}-a_{3} c_{3}\right), \\
\vartheta_{6}= & \Delta\left(b_{3} c_{3}+a_{4}^{2}\right)+\left(a_{2} c_{1}-a_{1} c_{2}\right)^{2} \\
& +2\left(a_{2} c_{1}-a_{1} c_{2}\right)\left(a_{4} c_{2}-a_{3} c_{3}\right), \\
\vartheta_{7}= & \Delta b_{2}+a_{2} a_{3}\left(c_{2}-c_{3}\right)+a_{1} a_{4}\left(c_{2}-c_{1}\right), \\
\vartheta_{8}= & \Delta b_{3}-\left(a_{3}^{2} c_{3}-2 a_{3} a_{4} c_{2}+a_{4}^{2} c_{1}\right), \\
\vartheta_{9}= & \operatorname{det}=\Delta\left(b_{2}^{2}-b_{1} b_{3}\right)+b_{1}\left(a_{3}^{2} c_{3}-2 a_{3} a_{4} c_{2}+a_{4}^{2} c_{1}\right) \\
& +2 b_{2}\left(a_{1} a_{4} c_{2}+a_{2} a_{3} c_{2}-a_{2} a_{4} c_{1}-a_{1} a_{3} c_{3}\right) \\
& +b_{3}\left(a_{2}^{2} c_{1}+a_{1}^{2} c_{3}-2 a_{1} a_{2} c_{2}\right)+\left(a_{1} a_{4}-a_{2} a_{3}\right)^{2}, \\
\vartheta_{10}= & b_{1} c_{1}+2 b_{2} c_{2}+b_{3} c_{3}+a_{1}^{2}+2 a_{2} a_{3}+a_{4}^{2} .
\end{aligned}
$$

Proposition 4. $S^{\prime}\left(\mathfrak{k}_{1}\right)^{H}$ is generated by the polynomials $c_{1}, c_{2}, c_{3}, \vartheta_{1}, \vartheta_{2}, \vartheta_{7}$, $\vartheta_{8}, \vartheta_{9}, \vartheta_{10}$.

Proof. A fastidious computation shows that the $\vartheta_{i}$ 's are invariants. (In the course of the proof, we will give some indications of how to get them.) Now for $\left(a_{i}, b_{j}, c_{k}\right)$ in a suitable open subset of $\mathfrak{k}_{1}$, the orbit $H\left(a_{i}, b_{j}, c_{k}\right)$ intersects the subspace given by $a_{1}=0, a_{2}=0, a_{3}=0$ at the point

$$
\left(0,0,0, c_{2}^{-1} \vartheta_{1}, c_{1}, c_{2}, c_{3}, \Delta^{-1} \vartheta_{2},\left(c_{2} \Delta\right)^{-1} \vartheta_{3},\left(c_{2}^{2} \Delta\right)^{-1} \vartheta_{4}\right) \text {. }
$$

Similarly, for $\left(a_{i}, b_{j}, c_{k}\right)$ in another open subset of $\mathfrak{k}_{1}$, the orbit $H\left(a_{i}, b_{j}, c_{k}\right)$ intersects the subspace given by $a_{1}=0, a_{2}=0, a_{4}=0$ at the point

$$
\left(0,0,-c_{3}^{-1} \vartheta_{1}, 0, c_{1}, c_{2}, c_{3}, \Delta^{-1} \vartheta_{2},\left(c_{1} c_{3} \Delta\right)^{-1} \vartheta_{5},\left(c_{3} \Delta\right)^{-1} \vartheta_{6}\right)
$$


Thus if $f \in S^{\prime}\left(\mathfrak{k}_{1}\right)^{H}$, there exists nonnegative integers $i, j, k, \ell, m$ such that

$$
\begin{array}{r}
c_{2}^{i} \Delta^{\prime} f \in \mathbb{C}\left[c_{1}, c_{2}, c_{3}, \vartheta_{1}, \vartheta_{2}, \vartheta_{3}, \vartheta_{4}\right], \\
c_{1}^{k} c_{3}^{\ell} \Delta^{m} f \in \mathbb{C}\left[c_{1}, c_{2}, c_{3}, \vartheta_{1}, \vartheta_{2}, \vartheta_{5}, \vartheta_{6}\right],
\end{array}
$$

and hence

$$
\Delta^{i+j+\ell+\ell+m} f \in \mathbb{C}\left[c_{1}, c_{2}, c_{3}, \vartheta_{1}, \vartheta_{2}, \vartheta_{3}, \vartheta_{4}, \vartheta_{5}, \vartheta_{6}\right] .
$$

But, as $\vartheta_{3}=c_{2} \vartheta_{7}, \vartheta_{4}=c_{1} \vartheta_{6}+\Delta \vartheta_{8}, \vartheta_{5}=c_{1} c_{3} \vartheta_{7}, \vartheta_{6}+c_{1} \vartheta_{2}+2 c_{2} \vartheta_{7}=\Delta \vartheta_{10}$, we have that for some nonnegative integer $n$ :

$$
\Delta^{n} f \in \mathbb{C}\left[c_{1}, c_{2}, c_{3}, \vartheta_{1}, \vartheta_{2}, \vartheta_{7}, \vartheta_{8}, \vartheta_{10}\right]
$$

Let us also remark that

$$
\vartheta_{7}^{2}-\vartheta_{2} \vartheta_{8}=\Delta \vartheta_{9}, \quad \vartheta_{1}^{2}+c_{1} \vartheta_{2}+2 c_{2} \vartheta_{7}+c_{3} \vartheta_{8}=\Delta \vartheta_{10}
$$

Now consider the obvious application

$$
\begin{aligned}
& \mathbb{C}\left[C_{1}, C_{2}, C_{3}, Y_{1}, Y_{2}, Y_{7}, Y_{8}, Y_{9}, Y_{10}\right] \\
& \quad \stackrel{\Phi}{\rightarrow} \mathbb{C}\left[c_{1}, c_{2}, c_{3}, \vartheta_{1}, \vartheta_{2}, \vartheta_{7}, \vartheta_{8}, \vartheta_{9}, \vartheta_{10}\right],
\end{aligned}
$$

(where the $C_{i}$ 's, $Y_{j}^{\prime}$ 's, are algebraically independent) and let us also introduce

$$
\begin{gathered}
\alpha: \mathbb{C}\left[a_{i}, b_{j}, c_{k}\right] \rightarrow \mathbb{C}\left[\theta, \omega, a_{i}, b_{j}\right] \\
c_{1} \mapsto \theta^{2}, c_{2} \mapsto \theta \omega, c_{3} \mapsto \omega^{2}, a_{i} \mapsto a_{i}, b_{j} \mapsto b_{j}
\end{gathered}
$$

It is not so difficult to see that $\operatorname{Ker} \alpha$ is the principal ideal expanded by $\Delta$. We claim that

$$
\operatorname{Ker}(\alpha \circ \Phi)=\left\langle C_{2}^{2}-C_{1} C_{3}, Y_{1}^{2}+c_{1} Y_{2}+2 c_{2} Y_{7}+c_{3} Y_{8}, Y_{7}^{2}-Y_{2} Y_{8}\right\rangle
$$

$\supseteq$ is clear. For $\subseteq$, let us introduce the auxiliary variables

$$
T_{1}=a_{4} \theta-a_{3} \omega, \quad T_{2}=-a_{2} \theta+a_{1} \omega .
$$

Let us observe that $\theta, \omega, T_{1}, T_{2}$ are linearly independent. Thus $\alpha \circ \Phi$ applies:

$$
\begin{array}{ll}
C_{1} \mapsto \theta^{2} & Y_{1} \mapsto-\theta T_{2}+\omega T_{1} \quad Y_{7} \mapsto T_{1} T_{2} \\
C_{2} \mapsto \theta \omega & Y_{2} \mapsto-T_{2}^{2} \quad Y_{8} \mapsto-T_{1}^{2} \\
C_{3} \mapsto \omega^{2} & Y_{9} \mapsto b_{1} T_{1}^{2}+2 b_{2} T_{1} T_{2}+b_{3} T_{2}^{2}+\left(a_{1} a_{4}-a_{2} a_{3}\right)^{2} \\
& Y_{10} \mapsto b_{1} \theta^{2}+2 b_{2} \theta \omega+b_{2} \omega^{2}+a_{1}^{2}+2 a_{2} a_{3}+a_{4}^{2} .
\end{array}
$$

Let $f \in \operatorname{Ker}(\alpha \circ \Phi)$. As the images of $Y_{9}, Y_{10}$ are linearly independent, we can assume that $f \in \mathbb{C}\left[C_{1}, C_{2}, C_{3}, Y_{1}, Y_{2}, Y_{7}, Y_{8}\right]$ and even that

$$
\begin{aligned}
f= & P_{1}\left(C_{1}, C_{2}, C_{3}, Y_{2}, Y_{7}, Y_{8}\right) \\
& +P_{2}\left(C_{1}, C_{2}, C_{3}, Y_{2}, Y_{7}, Y_{8}\right) Y_{1} .
\end{aligned}
$$

But the image of the first summand (resp., the second) is a sum of monomials of total degree in $\theta, \omega$ even (resp., odd) and hence

$$
\begin{aligned}
& P_{1}\left(C_{1}, C_{2}, C_{3}, Y_{2}, Y_{7}, Y_{8}\right) \\
& \quad=P_{2}\left(C_{1}, C_{2}, C_{3}, Y_{2}, Y_{7}, Y_{8}\right)=0,
\end{aligned}
$$

and the claim follows. 
Now we are ready to prove the proposition (i.e., that $\Phi$ is surjective). Let $f \in S^{\prime}\left(\mathfrak{k}_{1}\right)^{H}, n$ a nonnegative integer such that $\Delta^{n} f=\Phi(g)$ for some $g \in$ $\mathbb{C}\left[C_{1}, C_{2}, C_{3}, Y_{1}, Y_{2}, Y_{7}, Y_{8}, Y_{9}, Y_{10}\right]$. If $n=0$ we are done. If not, $g \in$ $\operatorname{Ker}(\alpha \circ \Phi)$ and hence (using $(*))$ :

$$
\Phi(g) \in \operatorname{Im} \Phi \Delta+\operatorname{Im} \Phi\left(\vartheta_{7}^{2}-\vartheta_{2} \vartheta_{8}\right)+\operatorname{Im} \Phi\left(\vartheta_{1}^{2}+c_{1} \vartheta_{2}+2 c_{2} \vartheta_{7}+c_{3} \vartheta_{8}\right),
$$
i.e.,

$$
\Phi(g) \in \operatorname{Im} \Phi \Delta .
$$

Thus $\Delta^{n-1} f \in \operatorname{Im} \Phi$ and the proposition follows.

Let us observe now that $\vartheta_{1}, \vartheta_{9}, \vartheta_{10}$ are $R$-invariants. Thus

$$
\begin{aligned}
S^{\prime}(\mathfrak{k})^{P} & \simeq\left(S^{\prime}\left(\mathfrak{k}_{1}\right)^{H} \otimes S^{\prime}\left(\mathfrak{k}_{2}\right)\right)^{R} \\
& \simeq \mathbb{C}\left[\vartheta_{1}, \vartheta_{9}, \vartheta_{10}\right] \otimes\left(\mathbb{C}\left[c_{1}, c_{2}, c_{3}, \vartheta_{2}, \vartheta_{7}, \vartheta_{8}\right] \otimes S^{\prime}\left(\mathfrak{k}_{2}\right)\right)^{R} .
\end{aligned}
$$

It is not so difficult to see that $\left\langle\vartheta_{2}, \vartheta_{7}, \vartheta_{8}\right\rangle$ is $R$-stable. Let us retain the notation of the above proposition. Considering

$$
\begin{aligned}
& \mathbb{C}\left[C_{1}, C_{2}, C_{3}, Y_{2}, Y_{7}, Y_{8}\right] \otimes S^{\prime}\left(\mathfrak{k}_{2}\right) \\
& \quad \rightarrow \mathbb{C}\left[c_{1}, c_{2}, c_{3}, \vartheta_{2}, \vartheta_{7}, \vartheta_{8}\right] \otimes S^{\prime}\left(\mathfrak{k}_{2}\right),
\end{aligned}
$$

and the following theorem by Formanek (see $[\mathrm{F}]$ ):

Theorem 10. If Ad denotes the irreducible $\mathrm{SL}(2, \mathbb{C})$-module of dimension 3, then $S^{\prime}(\operatorname{Ad} \oplus \operatorname{Ad} \oplus \mathrm{Ad})^{\mathrm{SL}(2, \mathbb{C})}$ is a hypersurface generated by the seven elements $\operatorname{tr}\left(X_{i} X_{j}\right), \operatorname{tr}\left(X_{1} X_{2} X_{3}\right)$ ( $X_{i}$ in the i-copy).

We can conclude

Theorem 11. $S^{\prime}(\mathfrak{k})^{P}$ is a hypersurface generated by 8 homogeneous polynomials $p_{1}, \ldots, p_{8}$ with degrees $2,2,2,2,2,4,4,5$ respectively, satisfying the relation:

$$
\begin{aligned}
p_{8}^{2}+ & p_{1} p_{7}^{2}-p_{2}\left(p_{3}^{2}-p_{4} p_{1}+p_{1}^{2}\right)^{2}+p_{1} p_{6} p_{5}^{2} \\
& +4 p_{1}^{2} p_{2} p_{6}+\left(p_{3}^{2}-p_{4} p_{1}+p_{1}^{2}\right) p_{5} p_{7}=0 .
\end{aligned}
$$

Proof. This follows from the explicit description of the generators in Formanek's theorem. Let us remark that a naive application of the quoted theorem will give 10 generators, but it is easy to reduce the number to 8 .

Proposition 5. $S^{\prime}(\mathfrak{g})^{K}$ and $S^{\prime}(\mathfrak{k})^{M}$ are hypersurfaces.

Proof. This follows from Theorem 11 (see [A2]).

Now we give a system of homogeneous generators for $S^{\prime}(\mathfrak{k})^{M}$. First of all, $S^{\prime}(\mathfrak{k})^{K}=S^{\prime}\left(\mathfrak{k}_{1}\right)^{K} \otimes S^{\prime}\left(\mathfrak{k}_{2}\right)^{K}$ is a polynomial ring generated by

$$
\begin{aligned}
& f_{1}=\operatorname{det}_{\mathfrak{k}_{2}}=\alpha^{2}+\beta \gamma, \\
& f_{2}=\operatorname{det}_{\mathfrak{k}_{1}}=\vartheta_{9}, \\
& f_{3}=a_{1}^{2}+a_{4}^{2}+2 a_{2} a_{3}+b_{1} c_{1}+2 b_{2} c_{2}+b_{3} c_{3} .
\end{aligned}
$$

We know from Remark 9 that $S^{\prime}\left(\mathfrak{k}_{1}\right)^{M}$ is a polynomial ring of Krull dim 4 ; indeed, it is generated by $f_{2}, f_{3}, f_{4}, f_{5}$, where

$$
f_{4}=a_{4}^{2}+b_{3} c_{3}, \quad f_{5}=a_{1}^{2}+b_{1} c_{1} .
$$


Obviously, $S^{\prime}\left(\mathfrak{k}_{2}\right)^{M}=S^{\prime}\left(\mathfrak{k}_{2}\right)^{K}$. So far, we need to find $f_{6}, f_{7}, f_{8}$, homogeneous of degree $2,4,5$ in $S^{\prime}(\mathfrak{k})^{M}$. This was done in [A2]. We get

$$
\begin{aligned}
f_{6}= & \left(\beta c_{1}+2 \alpha a_{1}+\gamma b_{1}\right) / 2, \\
f_{7}= & \beta\left(2 a_{3} a_{4} c_{2}+b_{3} c_{2}^{2}-a_{3}^{2} c_{3}\right) \\
& +2 \alpha\left(a_{2} a_{3} a_{4}-a_{4} b_{2} c_{2}+a_{2} b_{3} c_{2}+a_{3} b_{2} c_{3}\right) \\
& +\gamma\left(2 a_{2} a_{4} b_{2}+b_{2}^{2} c_{3}-a_{2}^{2} b_{3}\right) \\
f_{8}= & \beta\left(a_{2} a_{3} a_{4} c_{1}+a_{2} b_{3} c_{1} c_{2}+a_{1} a_{3}^{2} c_{3}+a_{3} b_{2} c_{1} c_{3}\right. \\
& \left.-2 a_{1} a_{3} a_{4} c_{2}-a_{4} b_{2} c_{1} c_{2}-a_{1} b_{3} c_{2}^{2}\right) \\
& +\alpha\left(-2 a_{2} a_{4} b_{2} c_{1}+a_{2}^{2} b_{3} c_{1}-a_{3}^{2} b_{1} c_{3}\right. \\
& \left.-b_{2}^{2} c_{1} c_{3}+2 a_{3} a_{4} b_{1} c_{2}+b_{1} b_{3} c_{2}^{2}\right) \\
& +\gamma\left(2 a_{1} a_{2} a_{4} b_{2}-a_{1} a_{2}^{2} b_{3}-a_{3} b_{1} b_{2} c_{3}+a_{1} b_{2}^{2} c_{3}\right. \\
& \left.+a_{4} b_{1} b_{2} c_{2}-a_{2} a_{3} a_{4} b_{1}-a_{2} b_{1} b_{3} c_{2}\right) .
\end{aligned}
$$

As their images in $S^{\prime}(\mathfrak{k})_{+}^{M} /\left(S^{\prime}(\mathfrak{k})_{+}^{M}\right)^{2}$ are linearly independent, $f_{1}, \ldots, f_{8}$ form a system of generators for $S^{\prime}(\mathfrak{k})^{M}$. The relation was found in [A2]:

Theorem 12. $S^{\prime}(\mathfrak{k})^{M}$ is generated by $f_{1}, \ldots, f_{8} ;$ the generating relation is

$$
\begin{aligned}
f_{8}^{2} & -f_{1}\left(f_{2}-f_{4} f_{5}-\frac{1}{4}\left(f_{3}-f_{4}-f_{5}\right)^{2}\right)^{2} \\
& -2\left(f_{2}-f_{4} f_{5}-\frac{1}{4}\left(f_{3}-f_{4}-f_{5}\right)^{2}\right) f_{6} f_{7} \\
& +f_{1}\left(f_{3}-f_{4}-f_{5}\right)^{2} f_{4} f_{5}-f_{5} f_{7}^{2} \\
& -\left(f_{3}-f_{4}-f_{5}\right)^{2} f_{4} f_{6}^{2}=0 .
\end{aligned}
$$

Furthermore, in order to get a system of generators of $S^{\prime}(\mathfrak{g})^{K}$ we need generators of $S^{\prime}(\mathfrak{k})^{M} \varphi_{1}, \ldots, \varphi_{8}$ such that $\varphi_{i} \in \bigoplus_{\gamma}: m(\gamma)=d_{i} S^{\prime}(\mathfrak{k})_{\gamma}^{M}$ for some $d_{i}$. In fact, we can deduce the $\varphi_{i}$ 's from the $f_{i}$ 's, decomposing the $\mathfrak{k}$-module generated by $f_{i}$ in irreducible components. We need too the $d_{i}$ 's; all this information is given below (see [A2] for the proofs):

$$
\varphi_{1}=f_{2}, \quad \varphi_{2}=f_{2}, \quad \varphi_{3}=\frac{1}{10} f_{3},
$$

of course with $d_{i}=0 \quad(i=1,2,3)$,

$$
\begin{aligned}
& \varphi_{4}=\frac{1}{2}\left(f_{4}-f_{5}\right) ; \quad d_{4}=2, \\
& \varphi_{5}=\frac{1}{2}\left(f_{4}+f_{5}-\frac{3}{5} f_{3}\right) ; \quad d_{5}=4, \\
& \varphi_{6}=f_{6} ; \quad d_{6}=2, \\
& \varphi_{8}=f_{8} ; \quad d_{8}=4, \\
& \varphi_{7}=f_{7}+\frac{1}{2} f_{6}\left(f_{3}-4 f_{4}\right), \quad d_{7}=2 .
\end{aligned}
$$

Therefore, a system of generators for $S^{\prime}(\mathfrak{g})^{K}$ is $\psi_{i}=\varphi_{i} \cdot H^{d_{i}}, i=1, \ldots, 8$, and $\psi_{9}=H^{2}$, where $H$ is a generator of $\mathfrak{a}^{*}$.

Finally, we want to give the relation between the $\psi_{j}$ 's. From Theorem 12 we obtain (after some cumbersome calculations; see [A2]):

Theorem 13. If $\mathfrak{g}_{\mathbb{R}}=\mathfrak{s p}(2,1) S^{\prime}(\mathfrak{g})^{K}$ is a hypersurface given by $\psi_{8}^{2}-4 \psi_{1} \psi_{2} \psi_{5}^{2}$ $-100 \psi_{1} \psi_{3}^{2} \psi_{5}^{2}-\psi_{1} \psi_{4}^{4}+20 \psi_{1} \psi_{3} \psi_{5} \psi_{4}^{2}+2 \psi_{6} \psi_{4}^{2} \psi_{7}-4 \psi_{2} \psi_{5} \psi_{6}^{2}-75 \psi_{3}^{2} \psi_{5} \psi_{6}^{2}-$ $10 \psi_{3} \psi_{4}^{2} \psi_{6}^{2}-10 \psi_{3} \psi_{6} \psi_{5} \psi_{7}+\psi_{9}\left(4 \psi_{2} \psi_{4} \psi_{6}^{2}-25 \psi_{3} \psi_{4} \psi_{6}^{2}+10 \psi_{3} \psi_{6} \psi_{4} \psi_{7}-\psi_{4} \psi_{7}^{2}\right)$ 
$+\psi_{9}^{2}\left(-2 \psi_{1} \psi_{2} \psi_{4}^{2}-700 \psi_{1} \psi_{3}^{3} \psi_{5}+70 \psi_{1} \psi_{3}^{2} \psi_{4}^{2}+28 \psi_{1} \psi_{2} \psi_{3} \psi_{5}-6 \psi_{3} \psi_{7}^{2}-2 \psi_{2} \psi_{6} \psi_{7}\right.$

$\left.-14 \psi_{2} \psi_{3} \psi_{6}^{2}-10 \psi_{3}^{2} \psi_{6} \psi_{7}+200 \psi_{3}^{2} \psi_{6}^{2}\right)+\psi_{9}^{4}\left(-\psi_{1} \psi_{2}^{2}-1225 \psi_{1} \psi_{3}^{4}+74 \psi_{1} \psi_{2} \psi_{3}^{2}\right)$ $=0$.

\section{REFERENCES}

[AG] O. M. Adamovich and E. O. Golovina, Simple linear groups having free algebra of invariants, Selecta Math. Soviet 3 (no. 2) (1983/1984).

[AP] E. M. Andreev and V. L. Popov, On stationary subgroups of points in general position in the Representation space of a Lie group, Funct. Anal. Appl. 5 (1971), 1-8.

[A1] N. Andruskiewitsch, A new proof of Tirao's restriction theorem Rev. Un. Mat. Argentina (to appear).

[A2] _ Computing some rings of invariants (to appear in the Proceedings of the IX ELAM).

[A3] _ $\ldots$, On the complicatedness of the pair $(\mathfrak{g}, K)$, Rev. Mat. Univ. Complut. Madrid 2 (1989).

[B] Abdel-Ilah Benabdallah, Generateurs de l'algebre $\mathfrak{U}(G)^{K}$ avec $G=\mathrm{SO}(m)$ ou $\mathrm{SO}_{\mathfrak{o}}(1, m-$ 1) et $K=\mathrm{SO}(m-1)$, Bull. Soc. Math. France 111 (1983), 303-326.

[Bo] N. Bourbaki, Algèbre commutative, Chapitres 3 et 4, Hermann, Paris, 1967.

[BT] A. Brega and J. A. Tirao, A property of a distinguished class of $K$-modules associated to the classical rank one semisimple Lie algebras, Proceedings of the IX ELAM (to appear).

[BLV] M. Brion, D. Luna, and Th. Vust, Espaces homogènes spheriques, Invent. Math. 84 (1986), 617-632.

[C] Allan Cooper, The classifying ring of groups whose classifying ring is commutative, Doctoral Thesis, MIT (unpublished).

[E] A. G. Elashvili, Canonical form and stationary subalgebras of points of general position for simple linear Lie groups, Funct. Anal. Appl. 6 (1972), 44.

[F] E. Formanek, Invariants and the ring of generic matrices, J. Algebra 89 (1984), 178-223.

[J] K. Johnson, The centralizer of a Lie algebra in an enveloping algebra, J. Reine Angew. Math. 395 (1989), 196-201.

[Kc] V. G. Kac, Some remarks on nilpotent orbits, J. Algebra 64 (1980), 190-213.

[K] B. Kostant, Lie group representations on polynomial rings, Amer. J. Math. 85 (1963), 327404.

[KPV] V. G. Kac, V. L. Popov, and E. B. Vinberg, Sur les groupes linéaires algébriques dont l'algèbre des invariantes est libré, C. R. Acad. Sci. Paris 283 (1976), 875-878.

[L] F. Levstein, On some rings of invariants that are polynomial rings, Proceedings of the IX ELAM (to appear).

[LR] D. Luna and R. W. Richardson, A generalization of the Chevalley restriction theorem. Duke Math. J. 46 (1979), 487-497.

[LV] D. Luna and T. Vust, Une théorème sur les orbites affines des groupes algébriques semisimples, Ann. Scuola Norm. Sup. Pisa Cl. Sci. 27 (1973), 527-535.

[Po] V. L. Popov, Representations with a free module of covariants, Funct. Anal. Appl. 10 (1977), 242-244.

[Po2] _ Stability criteria for the actions of a semisimple group on an algebraic manifold, Izv. Akad. Nauk SSSR Ser. Mat. 4 (1970), 527.

[Po3] _ The classification of representations which are exceptional in the sense of Igusa, Funct. Anal. Appl. 9 (1976), 348-350.

[Sch] G. W. Schwarz, Representations of simple Lie groups with regular rings of invariants, Invent. Math. 49 (1978), 167-191.

[Se] F. J. Servedio, Homogeneous vector spaces and varieties, Trans. Amer. Math. Soc. 176 (1973), 421-444. 
[T] J. Tirao, A restriction theorem for semisimple Lie groups of rank one, Trans. Amer. Math. Soc. 279 (1983), 651-660.

[VK] E. B. Vinberg and B. N. Kimel' feld, Homogeneous domains on flag manifolds and spherical subgroups, Funct. Anal. Appl. 12 (1978), 168-174.

Facultad de Matemática, Astronomia y Fisica, Valparaf́so y R. Martinez, 5000 Ciudad Universitaria, Córdoba, República Argentina

Current address: Mathematisches Seminar der Universität Hamburg, Bundesstrasse 55, 2000 Hamburg 13, Germany 